\section{Développement}

Le récepteur à activité tymosinekinase Neu/ErbB2/HER2 a d'abord été identifié et étudié pour son rôle dans le développement des tumeurs mammaires. Ses ligands, les neurégulines, ainsi que ErbB2 lui-même et des récepteurs de la même famille, jouent, en fait un rôle particulièrement important dans le développement du tissu nerveux, des cellules de Schwann et des jonctions neuromusculaires. Ce système est également impliqué dans la différenciation cardiaque, probablement dans les interactions inductrices à la base de la morphogenèse du cœur: Celle-ci nécessite, notamment, des signaux en provenance de cellules du système nerveux autonome dérivées de la crête neurale où sont exprimés des facteurs de transcription de la famille $b$ HLH, dénommés HAND. Des oligonucléotides anti-sens dirigés conjointement contre deux ARNm codant pour ces facteurs bloquent le développement cardiaque. Un autre exemple du jeu des influences inductrices dans la différenciation cellulaire et la morphogenèse est illustré par la différenciation du myotome qui pourrait nécessiter l'action combinée de Sonic hedgehog, produit par la notochorde, et des facteurs de la famille Wnt, produits par la partie dorsale du tube neural. Au niveau intestinal, une cellule souche des cryptes serait à l'origine de la différenciation des différents types cellulaires de l'épithélium de l'intestin grêle; la protéine à homéodomaine $C d x-2$ jouerait un rôle essentiel dans ces processus au cours desquels se succèdent des phénomènes d'activation et de répression de gènes.

\title{
Les neurégulines: une nouvelle famille de facteurs de croissance
}

La surexpression du proto-oncogène neu (ou erbB2 ou HER2) est liée au développement de nombre de tumeurs mammaires, ovariennes et gliales (revue dans [1]). Malgré l'intérêt suscité par ses propriétés oncogènes, ce récepteur transmembranaire à activité tyrosine kinase, proche parent du récepteur de l'EGF (epidermal growth factor), est longtemps resté orphelin. La quête de son ligand a conduit, en 1992, à l'identification des ADNc codant pour un groupe mosaïque de facteurs de croissance apparentés à l'EGF, les neurégulines, codés par les transcrits alternatifs d'un gène unique.

Les neurégulines sont connues sous des noms divers, attribués selon l'approche utilisée pour les isoler. La purification des protéines stimulatrices de l'activité tyrosine kinase de neu chez le rat a conduit à l'isolement de l'ADNc du NDF ou neu differentiation factor [2] et de ses homologues humains, les HRG ou hérégulines [3] possédant des effets différenciateurs et mitotiques sur des lignées de carcinomes mammaires. En parallèle, le clonage des ADNc codant pour des protéines identifiées sur la base de leur activité mitogène sur les cellules gliales du système nerveux central et périphérique, et sur leur effet sur la synthèse du récepteur de l'acétylcholine dans les myotubes, a conduit à l'isolement des ADNc des GGF (glial growth factors) et de l'ARIA (acetylcholine receptor inducing activity) $[4,5]$.

Des protéines sécrétées ou liées à la membrane

Les neurégulines correspondent, soit à des protéines sécrétées, soit à des protéines liées à la membrane et dont la partie extracellulaire peut être libérée par clivage protéolytique. Leurs précurseurs dérivent de l'épissage alternatif des treize exons d'un même gène $[2,4,6]$. La structure générale des précurseurs des neurégulines suit le schéma suivant : la partie extracellulaire aminoterminale est composée d'un peptide signal, d'un domaine immunoglobulinlike, d'un domaine glycosylé, d'un domaine EGF-like possédant six cystéines régulièrement espacées correspondant au domaine de liaison des récepteurs, d'un court domaine juxtamembranaire, d'un domaine transmembranaire et d'un domaine cytoplasmique (jigure 1). Ce schéma de base connaît des exceptions. L'absence des domaines transmembranaire et cytoplasmique pour certains GGF conduit, par exemple, aux formes sécrétées. L'absence de domaine de glycosylation, en particulier pour ARIA, ainsi que la présence additionnelle d'un domaine Kringle et/ou d'un domaine juxtamembranaire sont d'autres facteurs de variabilité. On connaît également plusieurs isoformes des neurégulines liées à des variations intrastructurales affectant les différents domaines. Ainsi, il existe deux variants du domaine EGF-like ( $\alpha$ et $\beta$ ), quatre variants du domaine juxtamembranaire (1 à 4) et trois variants du domaine cytoplasmique ( $\mathrm{a}, \mathrm{b}$ et $\mathrm{c}$ ). Certaines isoformes sont enrichies dans certains tissus. Les isoformes $\alpha 2$ et $\beta 2$ prédominent dans les cellules mésenchymateuses alors que l'isoforme $\beta 1$ se retrouve dans le système nerveux [7].

Bien que les neurégulines aient été d'abord identifiées sur la base de leur capacité d'activer ErbB2, des études 


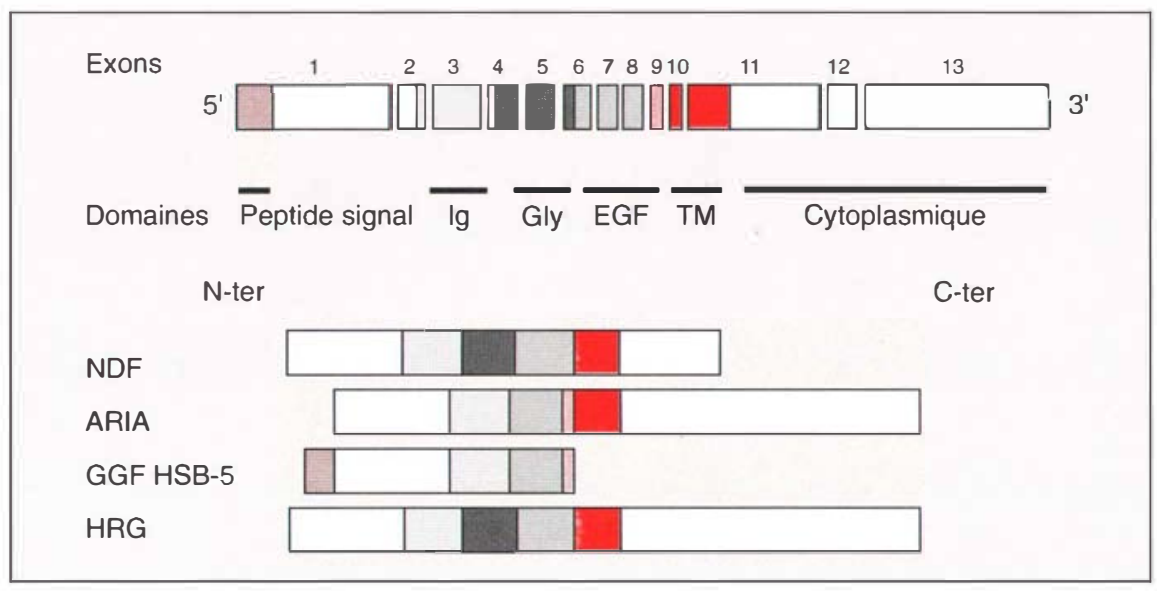

Figure 1. Structure schématique du gène neuréguline et de quelques-uns de ses produits. Ig: domaine immunoglobuline, Gly: domaine glycosylé, EGF: domaine epidermal growth factor like, TM: domaine transmembranaire.

ultérieures ont montré qu'elles ne se liaient pas directement à ce récepteur $[8,9]$. Deux autres récepteurs, membres de la famille de l'EGFR et capables de lier les neurégulines ont été récemment isolés: ErbB3 et

ErbB4 [10, 11]. Comme EGFR et Neu, ce sont des protéines transmembranaires possédant une activité tyrosine kinase intrinsèque dans leur domaine cytoplasmique [12] (figure 2). Les études menées à ce jour suggè-

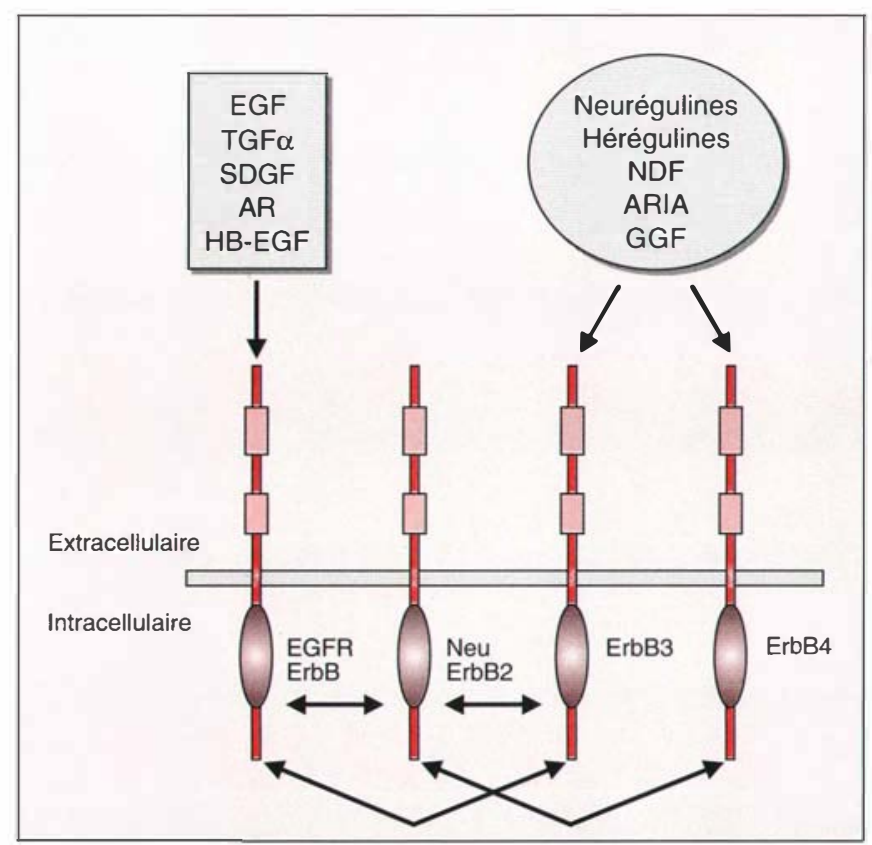

Figure 2. Les membres de la famille de l'EGFR, ou ErbB, et leurs ligands. L'ovale symbolise le domaine tyrosine kinase, les rectangles, les domaines riches en cystéines. Les flèches indiquent les hétérodimères dont le caractère fonctionnel est reconnu. EGF: epidermal growth factor, TGF $\alpha$ : transforming growth factor $\alpha, S D G F$ : schwannoma derived growth factor, AR: amphiréguline, HB-EGF: heparin binding-epidermal growth factor.

rent que ErbB3 et ErbB4 correspondent respectivement aux récepteurs à faible et forte affinité des neurégulines [13]. Mais, si la liaison des neurégulines à ErbB4 entraîne l'autophosphorylation du récepteur et une réponse biologique, il semble que l'activation d'ErbB3 dépende de la présence de ErbB2 [14]. De façon générale, la liaison du ligand à la partie extracellulaire de ces récepteurs entraîne leur homodimérisation mais aussi leur hétérodimérisation avec les autres membres de la famille de l'EGFR, y compris EGFR lui-même $[13,15]$. Cette combinatoire augmente la diversité potentielle des réponses liées à l'activation de ces récepteurs. En effet, la liaison du ligand au récepteur conduit à l'autophosphorylation des résidus tyrosine intracellulaires, points d'ancrage de substrats spécifiques assurant la transmission du signal [16]. Un même ligand peut ainsi entraîner des réponses cellulaires différentes selon la nature des récepteurs présentés par la cellule cible.

Des localisations variées mais prédominantes dans le système nerveux central

Les neurégulines se retrouvent aussi bien chez l'embryon que chez l'adulte dans le système nerveux central et périphérique ainsi que dans divers organes du parenchyme. Dans le système nerveux, les sources cellulaires des neurégulines ont été essentiellement localisées par hybridation in situ chez les rongeurs et l'homme, et montrent une très large répartition [17-19]. La plupart des neurones cholinergiques du système nerveux central expriment le gène des neurégulines tout au long de la vie embryonnaire et post-natale. Il s'agit notamment des motoneurones de la moelle épinière et du tronc cérébral, et des neurones cholinergiques du télencéphale basal. Mais les neurégulines se retrouvent également dans diverses structures non cholinergiques dont le cervelet, certains noyaux de l'hypothalamus et du thalamus ainsi que dans les zones subventriculaires des ventricules latéraux qui constituent une des zones germinatives d'où proviennent neurones et cellules gliales. Dans le système nerveux 


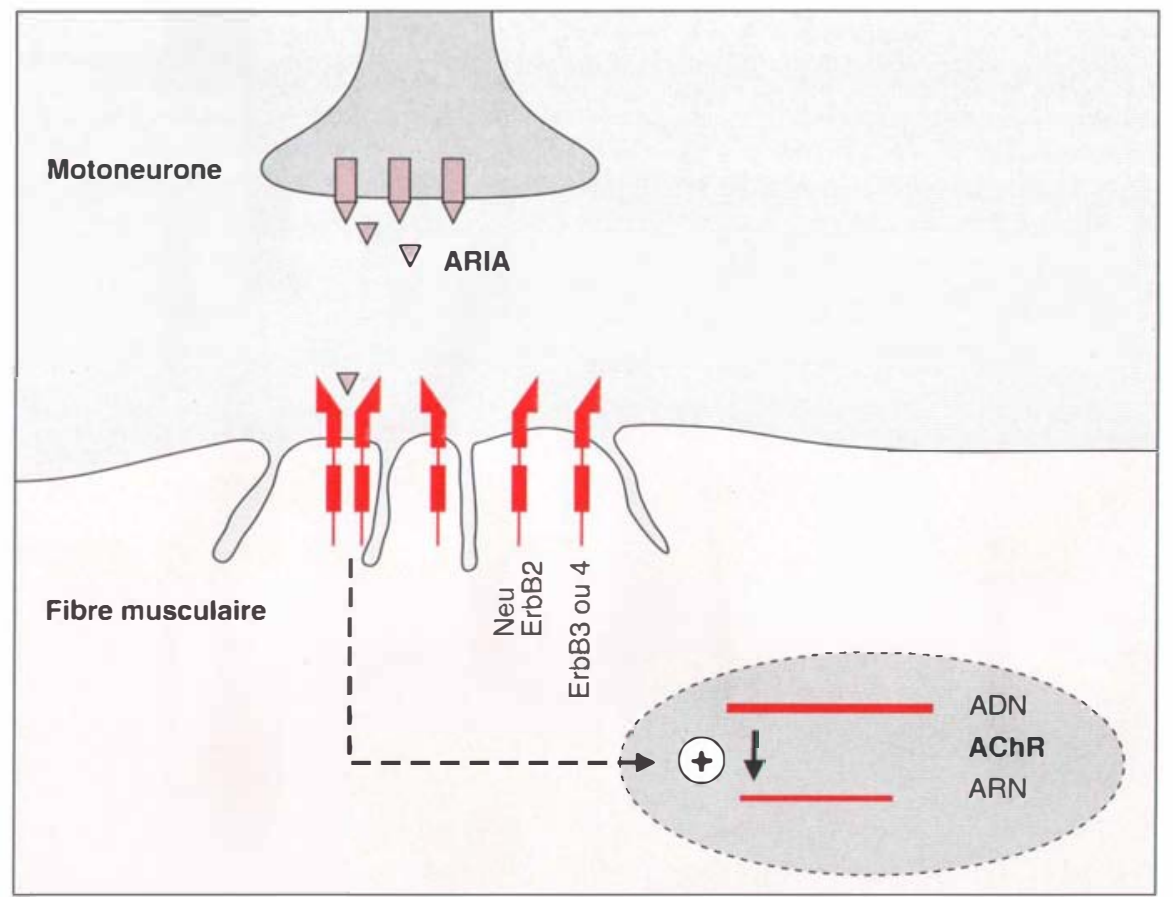

Figure 3. Rôle des neurégulines au niveau de la synapse neuromusculaire. ARIA (acetylcholine receptor inducing activity), I'un des produits du gène neuréguline, est synthétisé par le motoneurone et libéré au niveau de la fente synaptique où il se lie à ses récepteurs. La formation du complexe ligandrécepteur aboutit à la stimulation de la transcription du gène de l'AChR. II faut noter que les récepteurs peuvent former des hétérodimères fonctionnels.

périphérique, les neurégulines sont également synthétisées par les neurones sensoriels des ganglions des racines dorsales et des arcs branchiaux. Bien que les neurones constituent $a$ priori la source essentielle de neurégulines, les astrocytes semblent également capables de les synthétiser in vivo et in vitro [20].

En dehors du système nerveux, les neurégulines ont été retrouvées chez l'embryon dans des cellules d'origine mésenchymateuse du coeur, des poumons, des reins, des intestins, de l'estomac, des organes génitaux et dans les glandes mammaires [7, 21]. La synthèse des neurégulines persiste chez l'adulte, au moins dans le coeur. Enfin, les muscles embryonnaires synthétisent également les ARNm des neurégulines [18].

\section{Des messagers intercellulaires locaux}

L'examen des activités biologiques in vitro des neurégulines ainsi que la localisation de leurs récepteurs suggèrent que ces facteurs de croissance $\mathrm{m} / \mathrm{s} n^{\circ} 4$, vol. 12, avril 96

neurégulines, ARIA, est concentré au niveau des synapses neuromusculaires et dans la lame basale synaptique $\left(m / s n^{\circ} 3\right.$, vol. $11, p$. 493) [23, 24], tandis que les récepteurs ErbB2, 3 et 4 sont présents au niveau de la membrane musculaire post-synaptique $[25,26]$. In vitro, ARIA stimule la transcription des gènes codant pour les sous-unités de l'AChR [23, $25,27,28]$ ainsi que la synthèse des canaux sodiques musculaires [29]. L'ensemble de ces données suggère que les neurégulines constituent l'un des messagers motoneuronaux assurant la mise en place des synapses neuromusculaires (figure 3). L'élucidation de la contribution précise des neurégulines à l'établissement des jonctions neuromusculaires, ainsi que leur rôle chez l'adulte attend la production de souris chez lesquelles l'expression du gène de la neuréguline serait supprimée uniquement dans le système nerveux. En effet, les souris chez lesquelles le gène des neurégulines a été invalidé meurent au court de la première moitié de la vie embryonnaire (voir ci-dessous).

Les neurégulines ont été isolées, entre autres, sur la base de leur activité mitogène sur les cellules de Schwann qui forment la gaine de myéline des axones dans le système nerveux périphérique. Ces cellules dérivent de la crête neurale d'où elles migrent vers la périphérie le long des axones en croissance et leur prolifération dépend de leur contact avec l'axone [30, 31]. Morrissey et al. [32] ont montré au moyen de cocultures que le blocage à l'aide d'anticorps de la disponibilité des neurégulines produites par les neurones, ou des récepteurs ErbB2 présents sur les cellules de Schwann, supprimait les effets mitogènes des neurones sur ces dernières. Mais les neurégulines semblent également agir directement sur les précurseurs de la crête neurale en favorisant leur différenciation en cellules de Schwann [33].

Conséquences de l'invalidation du gène des neurégulines et des gènes de ses récepteurs

La publication de trois articles dans le numéro de novembre de Nature montrant les effets de la suppression 
des gènes codant pour les neurégulines, ErbB2 et ErbB4 a permis de préciser et d'étendre les fonctions des neurégulines au cours du développement embryonnaire malgré la mortalité précoce des mutants qui ne survivent pas au-delà du $11^{\mathrm{c}}$ jour de vie embryonnaire [34-36]. Les caractéristiques phénotypiques des mutants sont résumées dans le Tableau 1 . L'absence de précurseurs de cellules de Schwann le long des racines nerveuses de la moelle épinière chez les souris dont le gène des neurégulines a été invalidé [35] confirme le rôle central joué par ces facteurs dans les interactions au cours du développement entre les neurones et leur environnement glial, sans toutefois permettre de préciser si l'absence de neurégulines a affecté la migration, la différenciation ou la survie de ces cellules. Il en va de même pour les neurones ganglionnaires crâniens dérivés de la crête neurale dont le nombre est fortement réduit à la fois chez les mutants nuls de la neuréguline et de ErbB2. Le développement des nerfs moteurs crâniens qui dépend de la mise en place normale de leurs ganglions respectifs est également affecté [34, 35]. Les neurégulines semblent donc exercer un effet de type autocrine/paracrine sur les cellules de la crête neurale qui synthétisent à la fois les neurégulines et le récepteur ErbB2 au niveau du crâne. Au niveau du tronc, elles agissent d'abord sur le développement des précurseurs des cellules de Schwann, leurs effets passant par la mise en jeu de erbB2 et de erbB3 exprimés par ces dernières. En effet, la suppression de erbB4 ne modifie pas le développement des neurones ganglionnaires crâniens mais conduit à des voies d'innervation aberrantes du tronc cérébral [36]. Ce phénotype suggère d'abord que ErbB4 participe aux phénomènes de guidage des axones vers leurs cibles mais aussi qu'un ligand différent des neurégulines, qui reste à identifier, active ErbB4 à ce stade du développement du tronc cérébral.

La mortalité précoce des mutants nuls pour les neurégulines, erbB2 et erbB4 n'est cependant pas due aux altérations des phénotypes neuronaux

\begin{tabular}{|llll|}
\hline \multicolumn{4}{c|}{ Tableau I } \\
CARACTÉRISTIQUES PHÉNOTYPIQUES DES MUTANTS NULS \\
\hline & neurégulines-/- & erbB2-/- & erbB4-/- \\
\hline Létalité & E11 & E11 & E11 \\
\hline $\begin{array}{l}\text { Développement } \\
\text { des invaginations } \\
\text { du myocarde }\end{array}$ & avorté & avorté & avorté \\
\hline Cellules de Schwann & disparition & non déterminé & non déterminé \\
\hline $\begin{array}{l}\text { Nombre de neurones } \\
\text { ganglionnaires crâniens }\end{array}$ & réduit & réduit & normal \\
\hline Nerfs moteurs crâniens & $\begin{array}{l}\text { développement } \\
\text { altéré }\end{array}$ & $\begin{array}{l}\text { développement } \\
\text { altéré }\end{array}$ & $\begin{array}{l}\text { trajets } \\
\text { axonaux } \\
\text { aberrants }\end{array}$ \\
\hline
\end{tabular}

coeur. Dans les trois cas, on observe l'absence de développement des invaginations du myocarde qui amorcent la différenciation des ventricules. Les neurégulines sont synthétisées par les cellules de l'endocarde, tandis qu'ErbB2 et ErbB4 sont produits par les cellules du myocarde [35]. Bien que les effets de ces mutations n'aient pas été rapportés pour les autres organes du parenchyme, il est intéressant de noter que les transcrits des neurégulines ont été localisés chez. l'embryon de 10 jours dans les cellules mésenchymateuses du poumon qui entourent l'épithélium des bronchioles en formation [7]. Dans la glande mammaire, les neurégulines sont produites par les cellules mésenchymateuses chez la souris gestante et stimulent, dans des cultures organotypiques, la formation des alvéoles par les cellules épithéliales qui synthétisent ErbB3 et 4 [21].

Les neurégulines et leurs récepteurs apparaissent donc comme une famille complexe de facteurs de croissance dont les multiples effets dérivent notamment de leur rôle de médiateur dans des interactions paracrines entre deux types cellulaires différents. Si leur rôle au cours du développement embryonnaire précoce du système nerveux et du cour a pu être mis en lumière grâce à la production de mutations nulles, un nombre considérable de questions restent posées. Outre la fonction des neurégulines au cours de la deuxiè- me moitié de la vie embryonnaire dans le développement des divers organes du parenchyme qui synthétisent les neurégulines à cette période [7] et dans la mise en place des jonctions neuromusculaires, nous ne savons encore rien de leur rôle chez le jeune et chez l'adulte. Par ailleurs, la possibilité d'hétérodimérisation des récepteurs des neurégulines, notamment avec le récepteur de l'EGF dont les ligands sont souvent présents dans les mêmes tissus [37], permet d'envisager l'existence d'une "superfamille" de l'EGF dont les membres seraient susceptibles d'interagir de façon fonctionnelle. Enfin, bien que la découverte des neurégulines ait été stimulée par les propriétés transformantes du récepteur ErbB2, leur participation aux processus oncogènes reste un terrain vierge à explorer

\section{Marie-Pierre Junier Jean-Pierre Bellier}

Inserm U. 421, faculté de médecine, 8, rue du Général-Sarrail, 94010 Créteil, France.

\section{TIRÉS À PART}

M.P. Junier. 


\section{REFERENCES}

1. Stern DF, Hynes NE. The biology of ErbB-2/Neu/HER-2 and its role in cancer. Biochim Biophys Acta 1994 ; 1198 : 165-84.

2. Wen D, Peles E, Cupples R, Suggs SV, Bacus SS, Luo Y, Trail G, Hu S, Silbiger SM, Ben Levy R, Koski RA, Lu HS, Yarden Y. Neu differentiation factor: a transmembrane glycoprotein containing an EGF domain and an immunoglobulin homology unit. Cell $1992 ; 69$ : 559-72.

3. Holmes WE, Sliwskowski MX, Akita RW, Henzel WJ, Lee J, Park JW, Yansura D, Abadi N, Raab H, Lewis GD, Shepard HM, Kuang W], Wood WI, Goeddel DV, Vandlen RL. Identification of Heregulin, a specific activator of pl85 ${ }^{\text {abB2 }}$. Science $1992 ; 256$ : 1205-10.

4. Marchionni MA, Goodearl ADJ, Chen MS, Bermingham-McDonogh O, Kirk C, Hendricks M, Danehy F, Mismi D, Sudhalter J, Kobayashi K, Wroblewski D, Lynch C, Baldassare M, Hiles I, Davis JB, Hsuan JJ, Totty NF, Otsu M, McBurney RN, Waterfield MD, Stroobant P, Gwynne D. Glial growth factors are alternatively spliced ErbB2 ligands expressed in the nervous system. Nature 1993 ; 362 : 312-8

5. Falls DL, Rossen KM, Corfas G, Lane WS, Fishbach GD. ARIA, a protein that stimulates acetylcholine receptor synthesis is a member of the Neu ligand family. Cell $1993 ; 72: 801-5$.

6. Wen D, Suggs SV, Karunagaran D, Liu N Cupples RL, Luo Y, Janssen AM, Ben-Baruch N, Trollinger DB, Jacobsen VL, Meng SY, Lu HS, Hu S, Chang D, yang W, Yanigahara D, Koski RA, Yarden Y. Structural and functional aspects of multiplicity of neu differentiation factors. Mol Cell Biol 1994; 14: 1909-19.

7. Meyer D, Birchmeier C. Distinct isoforms of neuregulin are expressed in mesenchymal and neuronal cells during mouse development. Proc Natl Acad Sci USA 1994 ; 91 : 1064-8.

8. Peles E, Bacus SS, Koski RA, Lu HS, Wen $D$, Ogden SG, Ben Levy R, Yarden Y. Isolation of the Neu/HER-2 stimulatory ligand: a $44 \mathrm{Kd}$ glycoprotein that induces differentiation of mammary tumor cells. Cell 1992 ; 69 : 205-16.

9. Peles E, Ben-Levy R, Tzahar E, Liu N, Wen D, Yarden Y. Cell-type specific interaction of neu differentiation factors(NDF/heregulin) with neu/HER-2 suggests complex ligand-receptor relationships. EMBO J 1993 ; $3: 961-71$.

10. Kraus MH, Issing W, Miki T, Popescu NC, Aaronson SA. Isolation and characterization of ErbB3, a third member of the ErbB/epidermal growth factor receptor family: evidence for over expression in a subset of human mammary tumor. Proc Natl Acad Sci USA 1989 ; 86 : 9193-7.
11. Plowman DG, Culouscou JM, Withney GS, Green JM, Carlton GW, Foy L, Neubauer MG, Shoyab M. Ligand-specific activation of HER4/p180 $10 B 4$, a fourth member of the epidermal growth factor receptor family. Proc Natl Acad Sci USA 1993 ; 90 : 1746-50.

12. Peles E, Yarden Y. Neu and its ligands: from an oncogene to neural factors. BioEssays $1993 ; 15: 815-24$.

13. Tzahar E, Levkowitz G, Karunagaran D, Yi L, Peles E, Llavi S, Chang D, Liu N, Yayon A, Wen D, Yarden Y. ErbB-3 and ErbB-4 function as the respective low and high affinity receptors of all neu differentiation factor/heregulin isoforms. J Biol Chem 1994; $40: 25226-33$.

14. Beerly RR, Graus-Porta D, Woods-Cook K, Chen X, Yarden Y, Hynes NE. Neu differentiation' factor activation of ErbB-3 and ErbB-4 is cell specific and displays a differential requirement for ErbB-2. Mol Cell Biol $1995 ; 15: 6496-505$.

15. Wada T, Qian X, Greene MI. Intermolecular association of the $\mathrm{p} 185^{\text {new }}$ protein and EGF receptor modulates EGF receptor function. Cell $1990 ; 61: 1339-47$.

16. Ullrich A, Schlessinger J. Signal transduction by receptors with tyrosine kinase activity. Cell 1990 ; 61 : 203-12.

17. Chen MS, Bermingham-McDonogh $\mathrm{O}$ Danehy FT, Nolan C, Scherer SS, Lucas J, Gwynne D, Marchionni MA. Expression of multiple neuregulin transcripts in postnatal rat brain. J Comp Neurol 1994 ; $349: 389-400$.

18. Corfas G, Rossen KM, Aratake H, Krauss $R$, Fishbach GD. Differential expression of ARIA isoforms in the rat brain. Neuron $1995 ; 14: 103-15$

19. Ho WH, Armanini MP, Nuijens A, Phillips HS, Osheroff PL. Sensory and motor neuron-derived factor. I Biol Chem 1995; $270: 14523-32$.

20. Pinkras-Kramaski R, Eilam R, Spiegler O, Lavi S, Liu N, Chang D, Wen D, Schwart $M$, Yarden $Y$. Brain neurons and glial cells express neu differentiation factor/heregulin : a survival factor for astrocytes. Proc Natl Acad Sci USA 1994 ; 91 : 9387-91.

21. Yang $Y$, Spitzer E, Meyer D, Sachs M, Niemann C, Hartmann G, Weidner KM, Birchmeier C, Birchmeier W. Sequential requirement of hepatocyte growth factor and neuregulin in the morphogenesis and differentiation of the mammary gland. J Cell Biol $1995 ; 131$ : 215-26.

22. Burden SI. Synapse-specific gene expression. Trends Genet $1993 ; 9$ : 12-6.

23. Jo SA, Zhu X, Marchionni MA, Burden S]. Neuregulins are concentrated at nervemuscle synapses and activate ACh-receptor gene expression. Nature 1995 ; 373 : 158-61.
24. Sandrock AW, Goodearl ADJ, Yin OW, Chang D, Fishbach GD. ARIA is concentrated in nerve terminal at neuromuscular junctions and other synapses. I Neurosci $1995 ; 15$ : 6124-36.

25. Altiok N, Boisseau JL, Changeux JP. ErbB3 and ErbB2/neu mediate the effect of heregulin on acetylcholine receptor gene expression in muscle : differential expression at the endplate. $E M B O J 1995 ; 14$ : 4258-66.

26. Zhu X, Lai C, Thomas S, Burden SJ. Neuregulin receptor, ErbB3 and ErbB4, are localized at neuromuscular synapses. $E M B O$ $J 1995 ; 14: 5842-8$.

27. Harris DA, Falls DL, Dill-Devor RM, Fishbach GD. Acetylcholine receptor-inducing factor from chicken brain increases the level of mRNA encoding the receptor $\alpha$ subunit. Proc Natl Acad Sci USA 1988 ; 85 : 1983-7.

28. Martinou JC, Falls DL, Fischbach GD, Merlie JP. Acetylcholine receptor-inducing activity stimulates expression of the epsilonsubunit gene of the muscle acetylcholine receptor. Proc Natl Acad Sci USA 1991; 17: 7669-73

29. Corfas G, Fischbach GD. The number of $\mathrm{Na}^{+}$channels in cultured chick muscle is increased by ARIA, an acetylcholine receptorinducing activity. $J$ Neurosci 1993 ; 13 : 211825.

30. Zimmer C, Le Douarin N. Neural crest lineage. In: Dyck PJ, Thomas PK, Griffin JW, Low PA, Poduslo JF, eds. Peripheral neuropathy. Philadelphia: Saunders, 3d ed : 299316.

31. Wood PM, Bunge RP. Evidence that sensory axons are mitogenic for Schwann cells. Nature $1975 ; 256: 662-4$.

32. Morrissey TK, Levi ADO, Nuijens A, Sliwkowski MX, Bunge RP. Axon-induced mitogenesis of human Schwann cells involves heregulin and p185 erb2. Proc Natl Acad Sci USA 1995 ; 92 : 1431-5.

33. Shah NM, Marchionni MA, Isaacs I, Stroobant $\mathrm{P}$, Anderson DJ. Glial growth factor restricts mammalian neural crest stem cells to glial fate. Cell $1994 ; 77: 349-60$.

34. Lee KF, Simon H, Chen H, Bates B, Hung MC, Hauser C. Requirement for neuregulin receptor ErbB2 in neural and cardiac development. Nature $1995 ; 378$ : 394-8.

35. Meyer D, Birchmeier C. Multiple essential functions of neuregulin in development. Nature 1995 ; 378 : 386-90.

36. Gassmann M, Casagranda F, Orioli D, Simon H, Lai C, Klein R, Lemke G. Aberrant neural and cardiac development in mice lacking the ErbB4 neuregulin receptor. Nature $1995 ; 378: 390-4$.

37. Lee DC, Fenton SE, Berkowitz EA, Hissong MA. Transforming Growth Factor $\alpha$ : expression, regulation and biological activities. Pharmacol Rev $1995 ; 47: 51-85$. 


\section{BRĖVES}

Induction combinée de la myogenèse par Sonic hedgehog et des membres de la famille Wnt. Le mésoderme para-axial, adjacent au tube neural et à la notochorde, se divise au cours du développement en somites dont la partie ventrale forme le sclérotome, à l'origine des vertèbres et des côtes, et la partie dorsale le dermomyotome, à l'origine des muscles squelettiques et du derme. La différenciation du sclérotome semble être la conséquence de l'induction par la protéine Sonic hedgehog sécrétée par la notochorde et le plancher du tube neural [1]. En revanche, la différenciation des précurseurs des cellules musculaires semble nécessiter une induction combinée sous l'influence de la notochorde et d'autres régions du tube neural. Münsterderg et al. (Boston, MA, USA) démontrent maintenant que du mésoderme para-axial isolé d'embryons de poulets au stade 10 peut-être induit à exprimer des facteurs myogéniques de la famille MyoD par l'action combinée de cellules produisant le facteur Sonic hedgehog et des membres de la famille Wnt (Wnt-1, Wnt-3 et Wnt-4) [2]. Sonic hedgehog semble être le facteur inducteur produit par la notochorde et le plancher alors que les membres de la famille Wnt sont produits par la partie dorsale du tube neural. On ne peut déterminer, à partir des expériences réalisées, si l'action de Sonic Hedgehog est directe ou indirecte, c'est-àdire si ce facteur agit par lui-même ou bien en déclenchant la libération, par exemple par les cellules du sclérotome, d'un facteur myogénique. Un argument est une défaveur d'une action directe de Sonic Hedgehog sur la myogenèse: l'induction du sclérotome (et de son marqueur Pax-1) est observée pour des concentrations de Sonic Hedgehog plus faibles que l'induction de la myogenèse alors que le sclérotome est plus proche des sources de production de Sonic Hedgehog que le myotome.

[1. Concordet JP. médecine/sciences $1996 ; 12$ : 207-8.]

[2. Münsterderg AE, et al. Genes Dev 1995; 9 : 2911-22.]

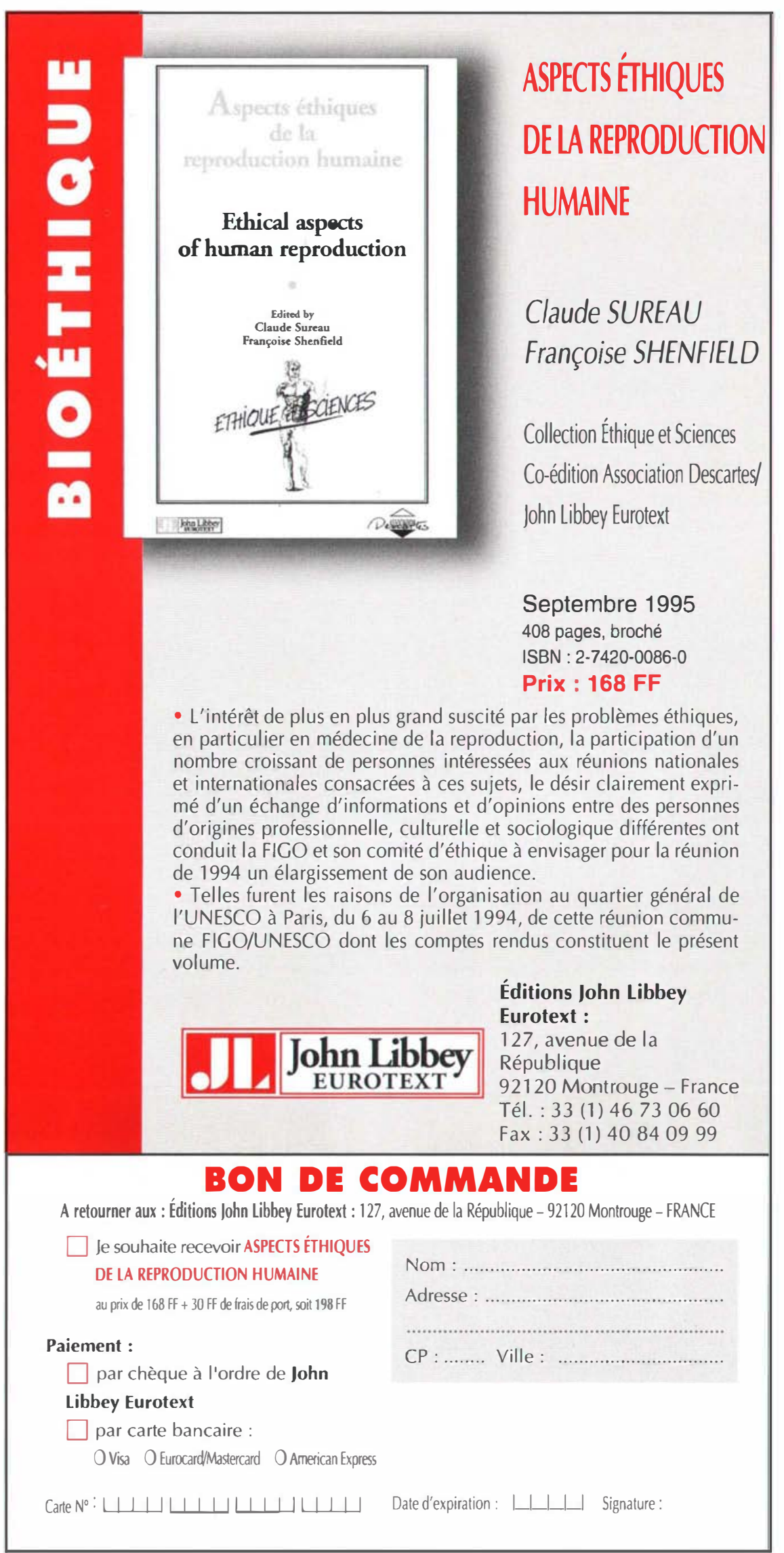

$\mathrm{m} / \mathrm{s} n^{\circ} 4$, vol. 12 , auril 96 